\title{
Descripción de las plantas medicinales de la familia Rubiacea con actividad biológica
}

\section{Description of the medicinal plants of the Rubiacea family with biological activity}

Lorenzo Mendoza Guzmán ${ }^{a}$, María del Carmen Valadez Vega ${ }^{b}$

\begin{abstract}
:
The Rubiaceae family is the fourth largest family in the plant kingdom, several species of this family have been used in traditional medicine, because it possesses anti-inflammatory, antiviral, anti-tumor, immunostimulant properties and others, the task of this review was to classify the species of the Rubiaceae family according to the most representative biological activity and to give a description of each classification.
\end{abstract}

\section{Keywords:}

Traditional medicine, Anti-inflammatory activity, anticarcinogenic, Rubiaceae

\section{Resumen:}

La familia Rubiaceae es la cuarta familia más grande dentro el reino de las plantas, varias especies de esta familia se han utilizado dentro de la medicina tradicional por poseer propiedades antiinflamatorias, antivirales, antitumorales, inmunoestimulantes, entre otras, el objetivo de esta revisión de bibliográfica fue clasificar a las especies de la familia Rubiaceae de acuerdo a su actividad biológica y dar una descripción de dichas actividades.

\section{Palabras Clave:}

Medicina tradicional, actividad antiinflamatoria, anticarcinogénico, Rubiaceae

\section{Introducción}

Las plantas han sido de gran utilidad para el ser humano desde el comienzo no solo por servir de alimento, sino también para curar o controlar enfermedades $(1,2)$.

En la actualidad se ha incrementado el interés en la medicina alternativa (plantas medicinales) para la cura de unos numerosos padecimientos y enfermedades que afectan a los seres humanos, por lo que las investigaciones que tengan como objetivo el cultivo, estudio y procesamiento de plantas medicinales con fines terapéuticos se consideran estratégicas importantes (35).
El conocimiento en el uso de plantas como una alternativa en la medicina, ha sido una práctica tradicional que se ha trasmitido generacionalmente a lo largo del tiempo (6). Se estima que al menos 35,000 especies de plantas presentan potencial para el uso médico y alrededor del $80 \%$ de la población mundial las utiliza como remedios tradicionales $(7,8)$.

La gama de plantas medicinales deriva de solo una fracción de la diversidad total, lo cual sugiere que no todas las plantas tienen presentes compuestos que les otorgan sus propiedades medicinales, por ello se debe de tener claramente definido el termino de planta medicinal, de acuerdo a la Organización Mundial de la Salud (OMS),

\footnotetext{
a Lorenzo Mendoza Guzmán, Universidad Autónoma del Estado de Hidalgo, México, https://orcid.org/0000-0001-9304-320X, Email: me308640@uaeh.edu.mx

${ }^{\mathrm{b}}$ Autor de Correspondencia, María del Carmen Valadez Vega, Universidad Autónoma del Estado de Hidalgo, México https://orcid.org/0000-
} 0001-6726-4113,Email: marynavaladez@ hotmail.com 
las plantas medicinales, como muchas otras especies de vegetales que contienen sustancias que pueden ser empleadas para propósitos terapéuticos o cuyos principios activos pueden servir de precursores para la síntesis de nuevos fármacos $(5,9,10)$.

Dentro de la amplia gama del reino de las plantas se encuentra la familia Rubiaceae, considerando su diversidad, es la cuarta más grande con unos 623 géneros y 10,200 especies (11).

México es un país megadiverso que se encuentra entre los países con mayor tradición ancestral y riqueza en el uso de plantas medicinales, debido a su amplia diversidad cultural (12), se han registrado alrededor de más de 4,000 especies para remedios tradicionales(13).

Dado a que se ha demostrado que la familia presenta actividad biológica, el objetivo de esta revisión es clasificar las propiedades medicinales de las especies de la familia Rubiaceae con base en su actividad biológica.

\section{Métodos}

Se realizó una revisión bibliográfica de las plantas de la familia Rubiacea con actividad biológica en las bases de datos como, PubMed, Google académico y Scielo, utilizando las palabras claves, familia Rubiacea, actividad biológica, fitoquímica, medicina tradicional, actividad anticancerígena, antiinflamatoria, antibacteriana y antioxidante. La revisión se llevó acabo en el periodo agosto-octubre de 2020.

\section{Resultados}

El resultado de la búsqueda entre las diferentes plataformas consultadas se muestra a continuación. Se revisaron un total de 60 artículos de los cuales se tomaron en cuenta los artículos en los que se mencionaban las siguientes palabras claves: actividad anticancerígena, antiinflamatoria, antibacteriana y antioxidante, obteniendo un total de 29 artículos citados en esta revisión.

El conocimiento de los pueblos indígenas ha sido primordial para la utilización de las plantas medicinales, el cual se ha trasmitido de generación en generación; lo que les ha permitido curar diversas enfermedades tales como: Afecciones en la piel, caída de cabello, caspa, fuegos, sarna, dolor de dientes y de cabeza; para enfermedades culturales como aire, espanto y mal de ojo, padecimientos del sistema circulatorio como anemia, circulación y presión arterial. Su utilización va desde terapias espirituales, platillos alimenticios, brebajes, cremas, infusiones y gotas $(9,14)$.

\section{Fitoquímica de la Familia Rubiaceae}

La familia Rubiaceae es una fuente primaria de productos naturales, medicinales, alucinógenos y venenos.
Diversos estudios químicos de alcaloides, encontraron tres tipos estructurales de alcaloides: indólicos, quinolínicos e isoquinolínicos (15). Varios géneros de Cinchoneae, especialmente la especie Cinchona officinalis $L$., contienen alcaloides tales como quinina, cinconidina, quinidina, cinconina, quinidina o quinotoxina y quinamina, empleados tradicionalmente en el tratamiento de desórdenes del ritmo cardíaco, fiebres, calambres musculares e indigestión $(15,16)$.

\section{Actividad anticancerígena}

Uncaria tomentosa, es un gran arbusto trepador crece formando enredaderas que alcanzan hasta veinte metros de altura, es nativa de Perú. Se han reportado estudios de actividad antitumoral. Los extractos acuosos de $U$. tomentosa inhibieron la proliferación de células HL60 y Raji, tuvo efecto de forma moderada en las células K562. El ácido ursólico aislado de esta planta tuvo efecto citotóxico en las líneas celulares SK MEL, KB, BT-549, SK-OV-3, y VERO con $\mathrm{Cl}_{50}$ entre 30 y $40 \mu \mathrm{g} / \mathrm{ml}$ en todas las líneas celulares (17).

También Uncaria rhynchophylla, presentó compuestos que inhibieron el crecimiento de las líneas celulares de cáncer A-549, HCT-15 y HT-1197, los esteres triperpenos tetracíclicos y el ácido uncarínocos inhibieron la fosfolipasa Cy1 (PLCY1) con $\mathrm{Cl}_{50}$ de 35.66 y $44.55 \mu \mathrm{M}$ respectivamente (17).

Otro estudio realizado por Bin Lim y Ryeol Lee en (2020) sobre Uncaria rhynchophylla (UR), evalúa la seguridad y la actividad biológica del extracto etanólico obtenido de esta planta. Se realizó extracción y fraccionamiento del extracto de UR obteniendo rendimientos del 6,71\%, 0,037\%, 0,042\%, 0,152\%, $0,332 \%$ y $5,132 \%$, para hexano, éter, DCM, EtOAC y fracciones acuosas, respectivamente. Se evaluó la seguridad de la mutagenicidad del extracto de UR, realizando ensayos de citotoxicidad e inducción de estrés oxidativo. El extracto de UR (2000 $\mu \mathrm{g}$ / placa) mostró una excelente actividad antimutagénica (superior al 90\%) contra BaP en las cepas TA98 y TA100. El extracto de UR exhibió DPPH eficiente (RC50 239,2 \pm 16,5 $\mu \mathrm{g} / \mathrm{ml}$ ) y actividad de atrapamiento del radical ABTS (RC 50 458,7 $\pm 25,0 \mu \mathrm{g} / \mathrm{ml})$. El extracto de UR $(150 \mu \mathrm{g} / \mathrm{mL})$ mostró actividad citoprotectora $(65,6 \% \pm 9,2 \%)$ contra $t$-BHP. Los extractos de $U$. rhynchophylla mostró una excelente actividad biológica a concentraciones no tóxicas, aunque la composición química de UR requiere de más investigaciones para un posible uso (18).

De Mitragyna inermes, el extracto metanólico $(\mathrm{MeOH})$ fue probado en las líneas celulares HL-60, Bel7402, 293 y KB, la fracción 1 presentó únicamente actividad citotóxica para la línea celular Bell-7402 
inhibiendo $>50 \%$ a una concentración de $10^{2} \mu \mathrm{g} / \mathrm{mL}$, esta fracción se purificó y se aisló el compuesto 3-O-( $\beta$-D6deoxy-glucopiranosil-ácidoquinóvico, obteniendo una inhibición del $43.01 \%$ y $91.74 \%$ el crecimiento de las células HeLa a una concentración $10^{2}$ y $10^{3} \mu \mathrm{g} / \mathrm{mL}$ (19).

C. alba fue evaluado en células Hep G2, RatH-4II-E, MDAMB-231, Hs 578T, 5637, teniendo mayor actividad citotóxica en la línea celular Hs 578T con un porcentaje de muerte celular del 100\%, en las líneas celulares Hep G2 y MDA-MB-231 no presentó actividad citotóxica y en la línea celular 5637 se obtuvo un $6.44 \%$ de muerte celular (20).

$H$. patens se evaluó en células Hep G2, RatH-4IIE, MDA-MB-231, Hs 578T, 5637, presentó un $10.56 \%$ de muerte celular en la línea celular MDA-MB-231 y en la línea celular Hep G2 no presentó actividad citotóxica (20). De igual manera, $P$. latifolia se evaluó en células Hep $G 2$, RatH-4II-E, MDA-MB-231, Hs 578T, 5637, obteniendo un $27.69 \%$ de células muertas en Hs $578 \mathrm{~T}$, mientras que en las líneas Hep G2 y MDA-MB-231 no tuvo efecto (20).

En otro estudio similar se evaluó la actividad citotóxica de los extractos crudos y sus fracciones de hojas y raíces de $G$. pohliana en nueve líneas celulares de cáncer humano: melanoma (UACC-62), mama (MCF-7), mama expresando el fenotipo de resistencia multifárma (NCl-ADR), pulmón (NCl-460), próstata (PCO-3), riñón (786-0), ovario (OVCAR), colon (HT-29) y leucemia (K562). La fracción de hexano de las hojas (HL) y las fracciones de acetato de etilo (EAR), cloroformo (CR) e hidrometanólico (HMR) de las raíces, fueron las fracciones más activas contra $\mathrm{K}-562$ con valores $\mathrm{DE} \mathrm{Cl}_{50}$ inferiores a $1 \mu \mathrm{g} / \mathrm{mL}(21)$.

De Heinsa crinita, se extrajeron dos nuevos triterpenoides de tipo ursano, 3 $\beta, 19 \alpha, 23$-trihidroxiurs-12-

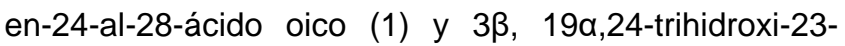
norurs-12-en-28- ácido oico (2), y ocho compuestos conocidos fueron aislados de las ramas y hojas. El ácido pomólico, $3 \beta, 12 \beta$-dihidroxi-5 $\alpha$-pregnano-16-es-20-uno y $12 \beta$-dihidroxi-5 $\alpha$-pregnano-16-es-3,20-diona mostraron citotoxicidad contra las líneas celulares HL-60, SMMC7721, A-549, MCF-7 y SW-480 con valores $\mathrm{IC}_{50}$ en el rango de 3.11-20.12 $\mu \mathrm{M}(22)$.

De extractos crudos de hojas secas de $S$. verticillata, obtenidos mediante extracción en metanol, se fraccionaron utilizando disolventes de creciente polaridad, el extracto y fracciones se evaluaron en la línea celular de cáncer de leucemia (MT-4). El extracto crudo mostró citotoxicidad con valores $\mathrm{Cl}_{50} 89 \mu \mathrm{g} / \mathrm{mL}$, y las fracciones en éter de petróleo y cloroformo mostraron valores $\mathrm{Cl}_{50} 74 \mu \mathrm{g} / \mathrm{mL}$ y $37 \mu \mathrm{g} / \mathrm{mL}$, respectivamente. Se demostró que los extractos crudos de $S$. verticillata presentan actividad citotóxica en la línea celular de la leucemia, determinando los valores MT-4 e IC 50 (23),
El estudio en Anthocephalus cadamba, fue diseñado para evaluar la actividad antioxidante y citotóxica de las fracciones de corteza e identificar sus compuestos activos. Se obtuvieron 4 fracciones de hexano (HACB), clorofromo (CACB), etilacetato (EACB) y n-butanol (NACB), se obtuvieron a partir del extracto crudo de metanol. Se encontró que NACB inhibió el crecimiento de células cancerosas de colon (COLO 205) con $\mathrm{Cl}_{50}$ de $54,36 \mu \mathrm{g} / \mathrm{mL}$ (24).

También se ha evaluado la actividad biológica del café, ya que es una bebida popular consumida en todo el mundo y las distintas variedades pertenecen a la familia Rubiaceae, por lo que se realizó el estudio de los extractos obtenidos de judías verdes y tostadas de Coffea canephora (Coffea canephora var. robusta), los extractos de café promovieron una disminución en la viabilidad celular, el ciclo celular modulado y la apoptosis inducida en la línea celular del carcinoma de próstata humana (DU-145) (25).

Se evalúo la citotoxicidad de Lasianthus trichophlebus en células neuroblastima SH-SY5Y, así como la actividad anti-amiloidogénica e inhibición de la ciclooxigenasa del extracto de metanol (LMT) y el extracto de cloroformo (LTC). Se determinó que ambos extractos mostraron inhibición citotóxica significativa contra las células SH-SY5Y con una Cl50 de 17.52 $\mathrm{mg} / \mathrm{mL}$ y $12.28 \mathrm{~g} / \mathrm{mL}$ respectivamente. El ensayo de tioflavina T indicó que la inhibición del extracto de LTC $(70,56 \%$ a $50 \mu \mathrm{g} / \mathrm{ml})$ era estadísticamente comparable $(p<0,05)$ al control positivo. La inhibición de la ciclooxigenasa contra las enzimas COX-1 y COX-2 dio Cl50 los valores para el extracto de LTM son 18,20 y $29,60 \mu \mathrm{g} / \mathrm{mL}$, respectivamente; mientras que el extracto de LTC mostró 4.11 y $2.78 \mu \mathrm{g} / \mathrm{mL}$, respectivamente. El estudio ha proporcionado información sobre la utilización de L. trichophlebus para desarrollar agentes vegetales más seguros para enfermedades antiinflamatorias 0 neurodegenerativas (26).

Galium verum, es una planta herbácea perenne, originaria de Europa y Asia, utilizada en la medicina tradicional como medicina contra el cáncer. En el estudio de Schmidt, et al. en 2014, obtuvieron el extracto de $G$. verum y lo aplicaron a líneas celulares de carcinoma de laringe quimiosencibles (Hep-2 y HLaC79) y quimiorecistentes que sobreexpresan la glicoproteína $P$ (Hep2 y Hep2-Tax, HLaC79-Tax), se demostró que el extracto acuoso de Galium es citotóxico para todas las líneas celulares. Realizando un análisis detallado de invasión 3D basado en esferoides de Hep2 y Hep2-Tax en geles de colágeno semisólidos y en diferentes recubrimientos de matriz extracelular, mostró una inhibición de la invasión por concentraciones subletales de decocción de Galium, lo cual demostró ser incluso más 
pronunciada en la línea celular Hep2-Tax quimiorresistente invasora más agresivamente (27).

De igual manera se realizó un estudio en Rubia yunnanensis es una planta medicinal que se cultiva al suroeste de china, en esa zona utilizan a esta planta para el tratamiento del cáncer; en el estudio se identificaron tres tipos principales de compuestos químicos, los ciclopéptidos, quinonas, triterpenos y el rubiarbonol (RG), es un triterpenoide único en $R$. yunnanensis, estos compuestos mostraron citotoxicidad en células cancerosas, pero no se ha mostrado el mecanismo de acción. RG mostró actividad citotóxica significativa en 7 líneas celulares de cáncer humano, cuya actividad se atribuyó a la inducción de apoptosis y detención de G0/G1 en células HeLa. Los resultados del estudio del mecanismo mostraron que $R G$ promovió la activación de la vía ERK1/2 y JNK en la familia MAPK, que a su vez aumentó la expresión de p53, desencadenando así la G0/G1 detención a través de la señalización p53/p21/ciclina D1 (28).

\section{Actividad antibacteriana}

Faramea occidentalis, se utiliza de forma tradicional como astringente, galactógeno y antiséptico. Se utilizó el extracto de las hojas de F. occidentalis para evaluar su actividad antibacteriana, el extracto seco mostró actividad antibacteriana frente a Salmonella typhimurium y Bacillus subtilis subsp spizizenii a $1240 \mu \mathrm{g} /$ disco; mientras que la concentración inhibitoria mínima para ambas cepas fue de $2048 \mu \mathrm{g} / \mathrm{mL}(29)$.

En la especie Isertia laevis, de la cual se evaluaron los extractos, fracciones y subfracciones de hoja sobre 18 cepas bacterianas pertenecientes a los géneros Streptococcus, Lactobacillus y Actinomyces, asociadas a la caries dental. El extracto en etanol y la fracción MeOH H20 mostraron actividad inhibitoria a 20 y $10 \mathrm{mg}$ sobre todas las cepas (30).

El estudio en Uncaria tomentosa evaluó el crecimiento de Staphylococcus aureus y Escherichia coli, la actividad antibacteriana se determinó mediante la técnica de difusión en agar en placas. Como resultado se observaron halos de inhibición en las 4 concentraciones al $50,100,500$ y $1000 \mu \mathrm{g} / \mathrm{mL}$ del extracto hidroalchohólico frente a Staphylococcus aureus, teniendo mayor halo de inhibición la concentración de la concentración de $50 \mu \mathrm{g} / \mathrm{mL}$ tuvo mayor halo de inhibición (14.3 $\mathrm{mm}$ ); sin embargo, no hubo inhibición en Escherichia coli (31).

Reportes han demostrado que Morinda citrifolia $L$ (noni) presenta actividad antimicrobiana frente a un gran número de bacterias y hongos patógenos. En un estudio realizado por Castillo et al. 2014, informa la actividad antimicrobiana in vitro de los extractos alcohólicos, hexánicos, clorofórmicos y de acetato de etilo de las hojas y semillas de la $M$. citrifolia $L$. (noni) frente a cepas de E. coli, S. aureus y Candida sp, obteniendo que los extractos evaluados de las hojas y semillas tuvieron actividad antimicrobiana frente a las cepas tratadas. De igual manera se realizó la identificación de compuestos, los principales metabolitos secundarios detectados fueron, en los extractos de hojas, quinonas, coumarinas y flavonoides; en las semillas, coumarinas y flavonoides respectivamente (32).

La especie Geophila repens, es una hierba perenne, de esta planta se han identificado compuestos del aceite esencial, en total se han identificado 77 compuestos, los cuales evaluaron su actividad antibacteriana del aceite esencial y también su efecto sinérgico con antibióticos convencionales. Los resultados demostraron que el aceite esencial de Geophila repens poseía una excelente actividad antibacteriana de amplio espectro, especialmente contra Pseudomonas aeruginosa y Bacillus subtilis. También demostró que la aplicación combinada del aceite con antibióticos obtuvo efectos sinérgicos en la mayoría de los casos; el efecto sinérgico más importante se notó cuando se combinó con estreptomicina y probado contra Escherichia coli (índices de concentración inhibitoria fraccional (FICl) de 0.13); por lo que la combinación del aceite y los antibióticos comerciales tiene un enorme potencial para el desarrollo de nuevos tratamientos (33).

Otra planta que se evaluó su actividad antimicrobiana es Gardenia coronaria, de esta planta se evaluó el extracto de metanol de las hojas el cual fue examinado para la estabilidad de la membrana y la actividad antibacteriana. Las concentraciones más bajas del extracto metanólico de hoja dieron buena actividad antimicrobiana y antiinflamatoria, pero las concentraciones más altas mostraron relativamente mayor actividad antibacteriana de proyección in vitro en comparación con Kanamicina. Los extractos metanolicos de hojas poseían una actividad antibacteriana de amplio espectro contra una variedad de organismos Gramnegativos y Grampositivos como Streptococcus agalactiae, Escherichia coli, Pseudomonas aeruginosa, Bacillus cereus, Shigella sonnei, Shigella boydii, y Proteus mirabilis, con una zona de inhibición de 10 a 1 $\mathrm{mm}$ (34).

\section{Actividad antiinflamatoria}

Palicourea crocea, es utilizada para tratar la inflamación (edema), el compuesto Croceanie A (PC-1) fue aislada de las hojas de $P$. crocea (MEPC), en un estudio por Samara et al. 2019, estudiaron su actividad antioxidante y antiinflamatoria. La actividad antioxidante se evaluó por 
DPPH, ABTS y MDA. MEPC (300, 100 y $300 \mathrm{mg} / \mathrm{kg})$ y PC-1 (10 y $30 \mathrm{mg} / \mathrm{kg}$ ) fueron probados para detectar efectos antiinflamatorios en edema de pata, pleuresía, sensibilidad al frío e hiperalgesia mecánica. Los resultados que se obtuvieron fueron para MEPC y alcaloide PC-1 demostraron un efecto antiedematogénico en edema de pata inducido por carragenina en 2 y $4 \mathrm{~h}$, también redujo significativamente la hiperalgesia mecánica, la respuesta en frío a la acetona en ratones, a 3 y $4 \mathrm{~h}$ después de la inyección, así como la migración de leucocitos en el modelo de pleuresía (35).

Otro estudio similar realizado por Álvares et al. 2014, evaluó cinco plantas medicinales, entre ellas Morinda citrifolia, perteneciente a la familia Rubiaceae, el método de evaluación de la inflamación fue por el modelo de edema de oreja inducido por aceite de Croton en ratones albinos OF-1 machos. La preparación de las decocciones de $M$. citriolia a $30 \%$, mostraron una reducción significativa del edema inducido en los ratones. El porcentaje de inhibición fue de $35.69 \%$, este puede clasificarse como leve, por lo cual el estudio da validación preclínica de la actividad antiinflamatoria de $M$. citrifolia (36).

Un estudio evaluó extractos de seis especies de la familia Rubiaceae (Psychotria capensis, Psychotria zombamontana, Pavetta lanceolata, Oxyanthus speciosus, Cremaspora triflora, Cephalanthus natalensis) combinadas con rifampicina para observar el efecto sinérgico in vitro en la actividad antiinflamatoria. La actividad antiinflamatoria se realizó mediante la inhibición de la producción de óxido nítrico (NO) utilizando macrófagos RAW 264.7 activados por LPS utilizando el ensayo Griess. Todos los extractos probados inhibieron la producción de óxido nítrico (NO) de manera dependiente de la concentración, con un porcentaje de inhibición que varía del 6,73 al 86,27\%(37).

Las hojas de W. heynei (familia: Rubiaceae) han sido utilizadas para tratar la hinchazón, heridas y dolores corporales. Por lo cual se evaluó el extracto de metanol de las hojas de $W$. heynei y las fracciones en ratas Sprague-Dawley. Los datos obtenidos mostraron que la fracción de acetato de etilo exhibió la mayor inhibición del edema en los modelos de edema auditivo inducido por TPA $(74.51 \pm 2.05)$ y edema anal inducido por aceite de crotón $(75.38 \pm 2.83)$. El pretratamiento con la fracción de acetato de etilo redujo el edema de la pata con formalina $(78.99 \pm 2.26 \%)$ después de $6 \mathrm{~h}$ y en carragenina $(75.71 \pm 4.46 \%)$ se detectó después de $4 \mathrm{~h}$. El nivel de marcadores antiinflamatorios; IL-1, IL-6, TNFo y PGE2 en el edema de pata inducido por carragenina en suero de rata fue significativamente $(p<0.001)$ disminuyó con el pretratamiento con la fracción de acetato etilo en la rata. Dado en los resultados se concluyó que las fracciones del extracto mostraron actividad antiinflamatoria (38).

La planta Pavetta indica (Familia: Rubiaceae) muestra un potencial antiinflamatorio del extracto de metanol, se evaluó contra varios modelos de inflamación como carragenina, histamina e inflamación inducida por dextrán en ratas. Los resultados mostraron que el extracto inhibió en un $48.41 \%, 41.10 \%$ y $24.22 \%$ respectivamente; en comparación con el control. El efecto se comparó con el fármaco indometacina estándar, es un fármaco antiinflamatorio no esteroideo estándar. El extracto de metanol también redujo de manera efectiva $y$ significativamente el granuloma inducido por pellet de algodón. El porcentaje de inhibición fue de 62.78 a la dosis de $500 \mathrm{mg} / \mathrm{kg}$, lo que sugiere su actividad en la fase proliferativa del proceso inflamatorio (39).

\section{Actividad antioxidante}

Un estudio realizado por Aro y Col, en 2016, evaluó la actividad antioxidante de los extractos de seis especies de la familia Rubiaceae (Psychotria capensis, Psychotria zombamontana, Pavetta lanceolata, Oxyanthus speciosus, Cremaspora triflora, Cephalanthus natalensis), en el cual se observó que los extractos de Psychotria zombamontana tuvieron mejor actividad antioxidante con un valor $\mathrm{IC}_{50}$ de $1,77 \mu \mathrm{g} / \mathrm{mL}$, inferior al valor $\mathrm{IC}_{50}$ de trolox y ácido ascórbico $(5.67 \mu \mathrm{g} / \mathrm{mL}$ y 4.66 $\mu \mathrm{g} / \mathrm{mL}$ respectivamente) (37).

Otro estudio similar, en Ixora parviflora, miembro de la familia de plantas con flores Rubiaceae rica en flavonoides y empleada como planta medicinal en la India; en el estudio se emplearon varios ensayos antioxidantes in vitro. Encontrando que el contenido fenólico total del extracto de Ixora parviflora (IPE) fue de $26.2 \mu \mathrm{g}$ equivalentes de ácido gálico (GAE)/mg de peso seco de las hojas y el contenido total de flavonoides fue de 54.2 a $4.4 \mu \mathrm{g}$ equivalentes de quercetina $(\mathrm{QE}) / \mathrm{mg}$, mientras que la concentración de ácido clorogénico fue de 9.7 a $1.2 \mathrm{mg} / \mathrm{g}$ del extracto. El IPE a $1000 \mu \mathrm{g} / \mathrm{ml}$ mostró una capacidad de reducción de 90.5 a $0.6 \%$, una actividad de barrido del radical 1,1-difenil-2-picrylhydrazy (DPPH) de 96.0 a $0.4 \%$, una actividad quelante ferrosa de 72.2 a $3.5 \%$, una actividad de barrido de energía del radical hidroxilo de 96.8 a $1.4 \%$, y una actividad de barrido de peróxido de hidrógeno de 99.5 a 3.3\%. Dado a los resultados obtenidos se demostró que IPE es un potente antioxidante, así como un agente potencial antifotoenvejecimiento (40).

El estudio de las hojas de Mitragyba speciosa en la evaluación de la actividad antioxidante mediante el método de barrido del radical DPPH, mostró que los valores de DPPH $\mathrm{IC}_{50}$ de los extractos acuosos, 
alcaloides y metanólicos fueron $213.4,104.81$ y 37.08 $\mu \mathrm{g} / \mathrm{mL}$, respectivamente. El contenido fenólico total de los extractos acuosos, alcaloides y metanólicos fue de 66,0 $\mathrm{mg}, 88,4,105,6 \mathrm{mg}$ de GAE/g, respectivamente, mientras que el total de flavonoides fue de 28.2, 20.0 y $91.1 \mathrm{mg}$ de $\mathrm{CAE} / \mathrm{g}$ respectivamente. Posteriormente se realizó una correlación entre la actividad antioxidante y el contenido fenólico total. El resultado sugirió que la actividad antioxidante relativamente alta del extracto metanólico en comparación con el extracto acuoso y alcaloide posiblemente podría ser a su alto contenido de fenoles (41).

El estudio de actividad antioxidante de Maytenus emerginata, en la cual se evaluó el extracto de las hojas por los métodos de DPPH, ABTS, óxido nítrico, ion superóxido y por el método quelante de hierro. También se estimó el contenido total de fenoles y flavonoides. Los valores de DPPH, ABTS, óxido nítrico, superóxido e $\mathrm{IC}_{50}$ quelante de hierro de los extractos metanólicos fueron 12.44, 24.27, 22.41, 5.85 y 2.74 $\mu \mathrm{g} / \mathrm{ml}$, respectivamente. El contenido fenólico total del extracto metanólico fue de $10.69 \mathrm{mg}$ de $\mathrm{CA} / \mathrm{g}$, mientras que el flavonoide total fue de $1.56 \mathrm{mg}$ de $\mathrm{CAE} / \mathrm{g}$, observando una correlación entre la actividad antioxidantes y el contenido fenólico total, lo que sugirió que la actividad antioxidante relativamente alta del extracto metanólico en comparación con el estándar (ácido ascórbico) podría ser debido a su alto contenido fenólico (42).

Scyphiphora hydrophyllacea es una planta de manglares en forma de arbustos de la familia Rubiaceae, el estudio realizado por Samarakoon et al. en 2017, evaluó la actividad antioxidante del extracto de las hojas, obteniendo que los ensayos de DPPH y ABST mostraron que el extracto metanólico tiene la mayor actividad de barrido de radicales libres. La actividad de barrido de radicales libres más baja fue en el extracto de acetato de etilo medido por el ensayo DPPH y en el extracto de hexano medido por el ensayo ABTS. Ambos ensayos mostraron una actividad moderada en el extracto de cloroformo (43).

\section{Conclusiones}

Dado a la gran diversidad y su amplia distribución a nivel mundial que se tiene de la familia Rubiaceae demuestra contener plantas con potencial citotóxico, antibacteriana, antiinflamatorio y antioxidante, lo cual fortalece su uso, ya que tiene un gran potencial para el tratamiento de diferentes tipos de enfermedades, por lo que se usó tradicional es importante para los pueblos indígenas así como los países subdesarrollados y en vías de desarrollo que en los últimos años han recurrido a las plantas para tratar enfermedades, siendo hoy en día avalada por la literatura científica, que ha demostrado que algunas plantas de esta familia presentan la actividad biológica que se les ha atribuido históricamente, dado a que en los últimos años se ha incrementado su empleo, ya que son de fácil acceso y la preparación es simple, ya que su uso va desde tés, infusiones, pastas, cremas, gotas, entre otras, por lo que su utilización es segura ya que los principios activos han demostrado ser inocuos en el organismo.

\section{Referencias}

1. Martínez-Moreno D, ... RA-F-B, 2006 undefined. Plantas medicinales de cuatro mercados del estado de Puebla, México. botanicalsciences.com.mx.

2. Villavicencio-Nieto MÁ. P-EBE. G-MAJ. Plantas tradicionalmente usadas como plaguicidas en el estado de Hidalgo, México. Polibotánica. 2010;30:193-238

3. Gómez-Dantés H, Lamadrid-Figueroa H, Cahuana-Hurtado L, Silverman-Retana O, Montero P, Cecilia González-Robledo M, et al. The burder of cancer in Mexico, 1990-2013. Vol. 58, Salud Publica de México. 2016.

4. Jacob PD, Buenaño AMP, Mancera RJ. Usos de plantas medicinales en la comunidad San Jacinto del Cantón Ventanas, Los Ríos - Ecuador. Rev UDCA Actual Divulg Científica. 2015 Jun 30;18(1):39-50.

5. Loraine S, Mendoza-Espinoza JA, Alberto J. Las plantas medicinales en la lucha contra el cáncer, relevancia para México. Rev Mex Ciencias Farm. 2010;41:18-27.

6. Pirondo A, Coulleri J, Keller H, Ferrucci M. Influencia de factores externos sobre la comercialización de plantas medicinales en un medio urbano: el caso de vendedores criollos e indígena en Corrientes, Argentina. 2011;

7. Annan K, Houghton PJ. Antibacterial, antioxidant and fibroblas growth stimulation of aqueous extracts of Ficus asperifolia Miq. and Gossypium arboreum L., wound-healing plants of Ghana. J Ethnopharmacol. 2008 Sep 2;119(1):141-4.

8. SOTERO GARCIA, A. I., ARTEAGA REYES, T. T., MARTINEZ CAMPOS, A. R., \& BUNGE VIVIER VE. Conocimiento local del género Arceuthobium en un Área Natural Protegida del centro de México. 2018 Mar 1;

9. Aguilar CA. Plantas medicinales del herbario IMSS : cuadros básicos por aparatos y sistemas del cuerpo humano. Instituto Mexicano del Seguro Social; 1994. 218 p.

10. Argueta A, Gallardo VMC. Atlas de las plantas de la medicina tradicional mexicana. Instituto Nacional Indigenista; 1994. 1786 p.

11. Borhidi A, Diego-Pérez N. Introducción a la taxonomía de la familia Rubiaceae en la flora de México. Acta Bot Hung. 2002 Jul 22;44(3-4):237-80.

12. Barragán-Solís A. La práctica de la autoatención por fitoterapia en un grupo de familias mexicanas. Arch en Med Fam. 2006;8(3):155-62.

13. Gómez Álvarez R. MEDICINAL PLANTS IN A SMALL VILLAGE IN THE STATE OF TABASCO, MEXICO. Rev Fitotec Mex. 2012;35(1):43-9.

14. Villaseñor RJL, Espinosa GFJ. Catálogo de malezas de México. Universidad Nacional Autónoma de México; 1998.

15. H García Barriga. Flora Medicinal de Colombia, Tomos I-III. sidalc.net. Colombia: Edit. Tercer Mundo.; 1992.

16. Meléndez-Gómez C, Kouznetsov V. ALCALOIDES QUINOLÍNICOS: IMPORTANCIA BIOLÓGICA Y 
ESFUERZOS SINTÉTICOS. Univ Sci. 2005;10:5-18.

17.

Heitzman ME, Neto CC, Winiarz E, Vaisberg AJ, Hammond GB. Ethnobotany, phytochemistry and pharmacology of Uncaria (Rubiaceae). Vol. 66, Phytochemistry. Pergamon; 2005. p. 5-29.

18.

Lim H Bin, Lee HR. Safety and biological activity evaluation of Uncaria rhynchophylla ethanolic extract. Drug Chem Toxicol. 2020;1-12.

Cheng ZH, Yu BY, Yang XW. 27-Nor-triterpenoid glycosides from Mitragyna inermis. Phytochemistry. 2002 Oct $1 ; 61(4): 379-82$.

Setzer, M. C., Moriarity, D. M., Lawton, R. O., Setzer, W. N., Gentry, G. A., \& Haber WA. Phytomedicinal potential of tropical cloudforest plants from Monteverde, Costa Rica. Rev Biol Trop. 2003;51:647-73.

21. De Oliveira PRN, Testa G, Medina RP, De Oliveira CMA, Kato L, Da Silva CC, et al. Cytotoxic activity of Guettarda pohliana Müll. Arg. (Rubiaceae). Nat Prod Res. 2013;27(18):1677-81.

22. Wu X De, He J, Li XY, Dong L Bin, Gong X, Gao X, et al. Triterpenoids and steroids with cytotoxic activity from Emmenopterys henryi. Planta Med. 2013;79(14):1356-61.

23. Ramcharan G, Clement YN, Maxwell AR. Cytotoxic activity of selected West Indian medicinal plants against a human leukaemia cell line. West Indian Med J. 2010 Dec;59(6):597601.

24. Chandel M, Kumar M, Sharma U, Singh B, Kaur S. Antioxidant, Antigenotoxic and Cytotoxic Activity of Anthocephalus cadamba (Roxb.) Miq. Bark Fractions and their Phytochemical Analysis using UPLC-ESI-QTOF-MS. Comb Chem High Throughput Screen. 2018 Apr 12;20(9):760-72.

25. Bauer D, Abreu J, Jordão N, da Rosa JS, Freitas-Silva O, Teodoro A. Effect of roasting levels and drying process of coffea canephora on the quality of bioactive compounds and cytotoxicity. Int J Mol Sci. 2018 Nov 1;19(11).

26. Tan MA, Lagamayo MWD, Alejandro GJD, An SSA. Neuroblastoma SH-SY5Y cytotoxicity, anti-amyloidogenic activity and cyclooxygenase inhibition of Lasianthus trichophlebus (Rubiaceae). 3 Biotech. 2020 Apr 1;10(4).

27. Schmidt M, Scholz CJ, Gavril GL, Otto C, Polednik C, Roller J, et al. Effect of Galium verum aqueous extract on growth, motility and gene expression in drug-sensitive and -resistant laryngeal carcinoma cell lines. Int J Oncol. 2014 Mar;44(3):745-60

Zeng GZ, Wang Z, Zhao LM, Fan JT, Tan NH. NF-кB and JNK mediated apoptosis and G0/G1 arrest of HeLa cells induced by rubiarbonol G, an arborinane-type triterpenoid from Rubia yunnanensis. J Ethnopharmacol. 2018 Jun 28;220:220-7.

29. Mijares LM, Galardis B, Yosvel M, Tamayo V, Yanelis Y, Tamayo A. Evaluación de la actividad antibacteriana del extracto seco de las hojas de Faramea occidentalis. Química Viva. 2014;13:164-73.

Alvarado Parada AC. Evaluación de la actividad inhibitoria de extractos, fracciones y subfracciones obtenidas de la planta Isertia Laevis sobre bacterias de importancia en caries dental. Rev Fed Odontol Colomb. 2008;8-15.

31. Moya Fernández WS. Efecto de diferentes concentraciones del extracto hidroalcohólico de uncaria tomentosa en el crecimiento de staphylococcus aureus y escherichia coli. Universidad Nacional de Trujillo. [Trujillo-Péru]: Universidad Nacional de Trujillo; 2017.

\section{Med. 2014;19(1):374-82.}

33. Rao H, Lai P, Gao Y. Chemical composition, antibacterial activity, and synergistic effects with conventional antibiotics and nitric oxide production inhibitory activity of essential oil from geophila repens (L.) I.M. Johnst. Molecules. 2017 Sep 1;22(9).

34. Chowdhury A, Azam S, Jainul MA, Faruq KO, Islam A. Antibacterial activities and in vitro anti-inflammatory (Membrane Stability) properties of methanolic extracts of gardenia coronaria leaves. Int J Microbiol. 2014;2014.

35. Formagio ASN, de Oliveira Junior PC, Volobuff CRF, Kassuya CAL, Ferreira DC, Cardoso CAL, et al. Anti-inflammatory Activity of Methanolic Extract and an Alkaloid from Palicourea crocea (Sw.) Roem and Schult. Inflammation. 2019 Jun 15;42(3):1045-55.

36. Brito Álvarez LG, Ana M, Frías Vázquez I, Francisco C, Morón Rodríguez J, Neyvis M, et al. Validación preclínica del efecto antiinflamatorio tópico de cinco plantas medicinales Preclinical validation of the topical anti-inflammatory effect of five medicinal plants. Rev Cuba Plantas Med. 2014;19(1):40-50.

37. Aro AO, Dzoyem JP, Eloff JN, McGaw LJ. Extracts of six Rubiaceae species combined with rifampicin have good in vitro synergistic antimycobacterial activity and good antiinflammatory and antioxidant activities. BMC Complement Altern Med. 2016 Oct 3;16(1).

38. Maryam S, Khan MR, Shah SA, Zahra Z, Batool R, Zai JA Evaluation of anti-inflammatory potential of the leaves of Wendlandia heynei (Schult.) Santapau \& Merchant in Sprague Dawley rat. J Ethnopharmacol. 2019 Jun 28;238.

39. Mandal SC, Lakshmi SM, Kumar CKA, Sur TK, Boominathan R. Evaluation of anti-inflammatory potential of Pavetta indica Linn. leaf extract (Family: Rubiaceae) in rats. Phyther Res. 2003 Aug;17(7):817-20.

40. Wen KC, Chiu HH, Fan PC, Chen CW, Wu SM, Chang JH, et al. Antioxidant Activity of Ixora parviflora in a Cell/Cell-Free System and in UV-Exposed Human Fibroblasts. Molecules. $2011 \mathrm{Jul} ; 16(7): 5735-52$.

41. Parthasarathy S, Azizi J Bin, Ramanathan S, Ismail S, Sasidharan S, Mohd MI, et al. Evaluation of antioxidant and antibacterial activities of aqueous, methanolic and alkaloid extracts from Mitragyna speciosa (rubiaceae family) leaves. Molecules. 2009 Oct;14(10):3964-74.

Gupta V, Sharma M. Phytochemical analysis and evaluation of antioxidant activities of methanolic extracts of Maytenus emarginata. Omi A J Integr Biol. 2012 May 1;16(5):257-62.

43. Samarakoon SR, Shanmuganathan C, Ediriweera MK, Piyathilaka P, Tennekoon KH, Thabrew I, et al. Antihepatocarcinogenic and anti-oxidant effects of mangrove plant Scyphiphora hydrophyllacea. Pharmacogn Mag [Internet]. 2017 Jan 1 [cited 2020 Oct 1];13(49):S76-83. Available from: https://pubmed.ncbi.nlm.nih.gov/28479730/ 